\title{
An improvement on the maximum number of $k$-Dominating Independent Sets
}

\author{
Dániel Gerbner $^{a} \quad$ Balázs Keszegh $^{a} \quad$ Abhishek Methuku $^{b}$ \\ Balázs Patkós ${ }^{a} \quad$ Máté Vizer ${ }^{a}$
}

November 6, 2018

${ }^{a}$ Alfréd Rényi Institute of Mathematics, HAS, Budapest, Hungary

H-1053, Budapest, Reáltanoda utca 13-15.

${ }^{b}$ Central European University, Budapest, Hungary

H-1051, Budapest, Nádor utca 9.

gerbner, keszegh, patkos@renyi.hu, abhishekmethuku, vizermate@gmail.com

\begin{abstract}
Erdős and Moser raised the question of determining the maximum number of maximal cliques or equivalently, the maximum number of maximal independent sets in a graph on $n$ vertices. Since then there has been a lot of research along these lines.

A $k$-dominating independent set is an independent set $D$ such that every vertex not contained in $D$ has at least $k$ neighbours in $D$. Let $m i_{k}(n)$ denote the maximum number of $k$-dominating independent sets in a graph on $n$ vertices, and let $\zeta_{k}:=$ $\lim _{n \rightarrow \infty} \sqrt[n]{m i_{k}(n)}$. Nagy initiated the study of $m i_{k}(n)$.

In this article we disprove a conjecture of Nagy and prove that for any even $k$ we have

$$
1.489 \approx \sqrt[9]{36} \leq \zeta_{k}^{k}
$$

We also prove that for any $k \geq 3$ we have

$$
\zeta_{k}^{k} \leq 2.053^{\frac{1}{1.053+1 / k}}<1.98,
$$

improving the upper bound of Nagy.
\end{abstract}

Keywords: independent sets, $k$-dominating sets, almost twin vertices AMS Subj. Class. (2010): 05C69 


\section{Introduction}

Let $G=G(V, E)$ be a simple graph. For any vertex $v \in V(G)$ let us denote by $d(v)$ the degree of $v, N(v)$ denotes the set of neighbors of $v$, also called the open neighborhood of $v$ and $N[v]$ denotes the closed neighborhood, i.e. $N[v]:=N(v) \cup\{v\}$.

A subset $I \subset V(G)$ is called independent if it does not induce any edges. A maximal independent set is an independent set which is not a proper subset of another independent set (that is, it cannot be extended to a bigger independent set). A subset $D \subset V(G)$ is a dominating set in $\mathrm{G}$ if each vertex in $V(G) \backslash D$ is adjacent to at least one vertex of $\mathrm{D}$, that is,

$$
\forall v \in V(G) \backslash D:|N(v) \cap D| \geq 1 .
$$

Erdős and Moser raised the question to determine the maximum number of maximal cliques that an $n$-vertex graph might contain. By taking complements, one sees that it is the same as the maximum number of maximal independent sets an $n$-vertex graph can have. A dominating and independent set $W$ of vertices is often called a kernel of the graph (due to Morgenstern and von Neumann [6]) and clearly, a subset $W$ is a kernel if and only if it is a maximal independent set.

The problem of finding the maximum possible number of kernels has been resolved in many graph families. To state (some of) these results, let $m i_{1}(n)$ denote the maximum number of maximal independent sets in graphs of order $n$, and let $m i_{1}(n, \mathcal{F})$ denote the maximum number of maximal independent sets in the $n$-vertex members of the graph family $\mathcal{F}$. Answering the question of Erdős and Moser, Moon and Moser proved the following well known theorem.

Theorem 1. (Moser, Moon, [5]) We have

$$
m i_{1}(n)= \begin{cases}3^{n / 3} & \text { if } n \equiv 0(\bmod 3) \\ \frac{4}{3} \cdot 3^{\lfloor n / 3\rfloor} & \text { if } n \equiv 1(\bmod 3) \\ 2 \cdot 3^{\lfloor n / 3\rfloor} & \text { if } n \equiv 2(\bmod 3)\end{cases}
$$

Moreover, they obtained the extremal graphs. If addition and multiplication by a positive integer denotes taking vertex disjoint union, then Moser and Moon proved that the equality is attained if and only if the graph $G$ is isomorphic to the graph $n / 3 K_{3}($ if $n \equiv 0(\bmod 3))$; to one of the graphs $(\lfloor n / 3\rfloor-1) K_{3}+K_{4}$ or $(\lfloor n / 3\rfloor-1) K_{3}+2 K_{2}($ if $n \equiv 1(\bmod 3))$; $\lfloor n / 3\rfloor K_{3}+K_{2}($ if $n \equiv 2(\bmod 3))$.

For the family of connected graphs the analogous question was raised by Wilf [11] and answered by the following result.

Theorem 2. (Füredi [2], Griggs, Grinstead, Guichard [3]) Let $\mathcal{F}_{\text {con }}$ be the family of connected graphs. Then

$$
m i_{1}\left(n, \mathcal{F}_{c o n}\right)= \begin{cases}\frac{2}{3} \cdot 3^{n / 3}+\frac{1}{2} \cdot 2^{n / 3} & \text { if } n \equiv 0(\bmod 3) \\ 3^{\lfloor n / 3\rfloor}+\frac{1}{2} \cdot 3^{\lfloor n / 3\rfloor} & \text { if } n \equiv 1(\bmod 3) \\ \frac{4}{3} \cdot 3^{\lfloor n / 3\rfloor}+\frac{4}{3} \cdot 3^{\lfloor n / 3\rfloor} & \text { if } n \equiv 2(\bmod 3)\end{cases}
$$


The extremal graphs are determined as well. In these graphs, there is a vertex of maximum degree, and its removal yields a member of the extremal graphs list of the previous theorem.

Wilf [11] and Sagan [10] investigated the case of trees and proved the following theorem.

Theorem 3. Let $\mathcal{T}$ be the family of trees. Then we have

$$
m i_{1}(n, \mathcal{T})= \begin{cases}\frac{1}{2} \cdot 2^{n / 2}+1 & \text { if } n \equiv 0(\bmod 2) \\ 2^{\lfloor n / 2\rfloor} & \text { if } n \equiv 1(\bmod 2)\end{cases}
$$

Hujter and Tuza determined the maximal number of kernels in triangle free graphs by proving the following result.

Theorem 4. ([4]) Let $\mathcal{T}_{\Delta}$ be the family of triangle-free graphs. Then for any integer $n \geq 4$ we have

$$
m i_{1}\left(n, \mathcal{T}_{\Delta}\right)= \begin{cases}2^{n / 2} & \text { if } n \equiv 0(\bmod 2) \\ 5 \cdot 2^{(n-5) / 2} & \text { if } n \equiv 1(\bmod 2)\end{cases}
$$

Other related results can be found in the survey of Chang and Jou [1].

There are lots of variants of domination studied in the literature. A quite natural and often considered one is $k$-domination. A set $D$ is called $k$-dominating if each vertex in $V(G) \backslash D$ is adjacent to at least $k$ vertices of $D$. In other words,

$$
\forall v \in V(G) \backslash D:|N(v) \cap D| \geq k
$$

A $k$-dominating independent set is called a $k$-DIS for short. Note that 1-DISes are exactly maximal independent sets. This notion was introduced by Włoch [12]. Nagy [7, 8] addressed the problem of determining the maximum number of $k$-dominating independent sets (for a given $k \geq 2)$ in an $n$-vertex graph. Generalizing $m i_{1}(n)$ and $m i_{1}(\mathcal{F})$ we introduce the following notation.

Notation 5. For $n, k \geq 1$ let $m_{k}(n)$ denote the maximum number of $k$-DISes in graphs of order $n$, and let $m i_{k}(n, \mathcal{F})$ denote the maximum number of $k$-DISes in an n-vertex graph from the family $\mathcal{F}$. If $\mathcal{F}$ consists of a single graph $G$, we denote by $i_{k}(G)$ the number of $k$-DISes in $G$.

In [8] Nagy proved that for all $k \geq 1$

$$
\zeta_{k}:=\lim _{n \rightarrow \infty} \sqrt[n]{m i_{k}(n)}
$$

exists. Theorem 1 implies $\zeta_{1}=\sqrt[3]{3}$ and, by definition, for $k \geq 2$ we have $\zeta_{k} \in[1, \sqrt[3]{3}]$. The following upper and lower bounds were established on the values of $\zeta_{k}$. 
Theorem 6. (Theorem 1.7 [8]) For all $k \geq 3$ we have:

$$
\sqrt{2} \leq \zeta_{k}^{k} \leq 2^{\frac{k}{k+1}}
$$

Theorem 7. (Theorem 1.6 [8]) We have

$$
1.489 \approx \sqrt[9]{36} \leq \zeta_{2}^{2} \leq \sqrt[5]{9} \approx 1.551
$$

Nagy conjectured in [8] (Conjecture 2, p19) that the lower bound of Theorem [6 will be the value of $\zeta_{k}^{k}$. Our following theorem disproves this conjecture.

Theorem 8. For any even $k$ we have

$$
\sqrt[9]{36} \leq \zeta_{k}^{k}
$$

Furthermore, $\lim _{\infty} \zeta_{k}^{k}$ exists and is at least $\sqrt[9]{36}$.

In this paper, our aim is to show that there is a constant $\eta>0$ such that $\zeta_{k}^{k}<2-\eta$ for all $k \geq 3$, thus improving Theorem 6 .

Theorem 9. For $k \geq 3$ we have

$$
\zeta_{k}^{k} \leq 2.053^{\frac{1}{1.053+1 / k}}<1.98
$$

Remark 10. It is easy to see that $1.98<2^{k /(k+1)}$ for $k \geq 588503$. In fact, the following calculation shows that Theorem 9 improves Theorem 6 for all $k \geq 3$. We want to show that

$$
2^{k /(k+1)}>(2+\varepsilon)^{1 /(1+\varepsilon+1 / k)},
$$

for $\varepsilon=0.053$ and any $k \geq 3$. After rearranging we get

$$
2^{\varepsilon}>(1+\varepsilon / 2)^{1+1 / k}
$$

which is true for $\varepsilon=0.053$ and $k=3$. Therefore, it is true for any larger $k$.

The remainder of the paper is organized as follows. In Section 2 we prove Theorem 8 , in Section 3 we prove Theorem 9 and we finish the article with some remarks and open questions in Section 4.

\section{Constructions - Proof of Theorem 8}

In this section we gather some observations that are related to lower bound constructions. To be more formal, we introduce the following function: let $m(k, t)$ denote the smallest integer $n$ such that there exists a graph on $n$ vertices that contains at least $t k$-DISes. For our constructions we will need two types of graph products: the lexicographic product $G \cdot H$ 
of two graphs $G$ and $H$ has vertex set $V(G) \times V(H)$ and any two vertices $(u, v)$ and $(x, y)$ are adjacent in $G \cdot H$ if and only if either $u$ is adjacent with $x$ in $G$ or $u=x$ and $v$ is adjacent with $y$ in $H$.

The cartesian product $G \times H$ of two graphs $G$ and $H$ also has vertex set $V(G) \times V(H)$ and any two vertices $(u, v)$ and $(x, y)$ are adjacent in $G \cdot H$ if and only if both $u$ is adjacent with $x$ in $G$ and $v$ is adjacent with $y$ in $H$.

All our lower bounds follow from the following remark.

Proposition 11. For any positive integers $k, l, t$, we have

(i) $m i_{k}(n) \geq t^{\left\lfloor\frac{n}{m(k, t)}\right\rfloor}$, and

(ii) $m(k l, t) \leq \operatorname{lm}(k, t)$.

Proof. To prove (i) observe that if $G$ is a graph on $m(k, t)$ vertices containing at least $t$ $k$-DISes, then the graph $G^{\prime}$ consisting of $\left\lfloor\frac{n}{m(k, t)}\right\rfloor$ disjoint copies of $G$ and possibly some isolated vertices, contains at least $t^{\left\lfloor\frac{n}{m(k, t)}\right\rfloor}$ many $k$-DISes. Indeed, all isolated vertices must be contained in every $k$-DIS of $G^{\prime}$, and to form a $k$-DIS of $G^{\prime}$, one has to pick a $k$-DIS in every copy of $G$.

To prove (ii) let $G$ be a graph on $m(k, t)$ vertices containing at least $t k$-DISes. Then, if we denote by $E_{l}$ the empty graph on $l$ vertices, the graph $G^{\prime}=G \cdot E_{l}$ has $\operatorname{lm}(k, t)$ vertices and if $I$ is a $k$-DIS in $G$, then $I^{\prime}=\{(u, v): u \in I\}$ is a $(k l)$-DIS in $G^{\prime}$.

Proof of Theorem 8 . First note (as observed by Nagy already) that $K_{3} \times K_{3}$ contains 6 2-DISes on 9 vertices. Therefore, by (ii) of Proposition 11, for every even $k$ we have

$$
m(k, 6) \leq \frac{k}{2} m(2,6) \leq \frac{9 k}{2}
$$

Part (i) of Proposition 11 yields the statement for even $k$.

Proposition 12. $m(k, 2)=2 k, m(k, 3)=3 k$.

Proof. The upper bounds are given by $K_{k, k}$ and $K_{k, k, k}$. For the lower bounds, note that if $A$ and $B$ are two different $k$-DISes, then we have $|A \backslash B| \geq k$ and $|B \backslash A| \geq k$. Indeed, e.g., if $v \in A \backslash B$ then $N(v)$ must contain at least $k$ vertices in $B$, while none of these are in $A$. This observation immediately shows we need at least $2 k$ vertices for $2 k$-DISes. One can easily see by analyzing possible intersection sizes that it also shows we need at least $3 k$ vertices for $3 k$-DISes.

Note that $K_{k, k, \ldots, k}$ gives $m(k, t) \leq t k$. Nagy [8] showed $m(2,4)=8$ and $m(2,6)=9$.

\section{Proof of Theorem 9}

First of all we fix $k \geq 3$. Let $\varepsilon=0.053$ and choose $c$ such that

$$
c^{k}=(2+\varepsilon)^{\frac{1}{1+\varepsilon+1 / k}} .
$$


We need to show that $m i_{k}(n) \leq A c^{n}$ for some absolute constant $A$. We will proceed by induction on $n$ and the base case is covered by a large enough choice of $A$. Let $G$ be a graph on $n$ vertices containing maximum possible number of $k$-DISes. We assume that every vertex belongs to at least one $k$-DIS, as otherwise we can delete the vertex without decreasing the number of $k$-DISes. Let $v$ be a vertex of minimum degree in $G$ that we denote by $\delta$. Note that we may assume $\delta \geq k$. Indeed, if a vertex $v$ has degree less than $k$, then it is easy to see that it must be contained in every $k$-DIS of $G$. Then it follows that the number of $k$-DISes in $G$ is at most $m i_{k}(n-|N(v)|-1$ ) (where $N(v)$ denotes the set of vertices adjacent to $v$ ) and we are done by induction.

Consider the following two cases:

Case 1: $\delta \geq(1+\varepsilon) k$.

In this case we use Proposition 5.1 from [8]. Following an inductive argument of Füredi [2], Nagy proved that we have

$$
m i_{k}(n)=m i_{k}(G) \leq c_{0} \max _{\delta \in \mathbb{Z}^{+}}\left\{\left(\frac{k+\delta}{k}\right)^{\frac{n}{\delta+1}}\right\} .
$$

for some universal constant $c_{0}$. Let $\delta=\left(1+\varepsilon^{\prime}\right) k$. Then we have

$$
m i_{k}(n) \leq c_{0}\left(2+\varepsilon^{\prime}\right)^{\frac{1}{1+\varepsilon^{\prime}+1 / k} \frac{n}{k}}
$$

By Proposition 14 (see Appendix), the right hand side of the above inequality is monotone decreasing in $\varepsilon^{\prime}$. Since $\delta \geq(1+\varepsilon) k$, we have $\varepsilon^{\prime} \geq \varepsilon$. So for fixed $k \geq 3$ we conclude that

$$
m i_{k}(n) \leq c_{0}(2+\varepsilon)^{\frac{1}{1+\varepsilon+1 / k} \frac{n}{k}}=O\left(c^{n}\right) .
$$

Case 2: $\delta \leq(1+\varepsilon) k$.

In this case we combine the inductive argument with a new idea. Let $v$ be a vertex of degree $\delta$. The number of $k$-DISes containing $v$ is at most $m i_{k}(n-\delta-1)$ and to bound the number of $k$-DISes not containing $v$, we introduce the following auxiliary graph. We say that two non-adjacent vertices $x, y$ of $G$ are almost twins if

$$
|N(x) \backslash N(y)|,|N(y) \backslash N(x)|<k
$$

hold. We define $T_{G}$ to be the graph with vertex set $N(v)$ and $x, y$ form an edge in $T_{G}$ if they are almost twins in $G$.

Proposition 13. If $x, y$ belong to the same connected component in $T_{G}$, then they belong to the same $k$-DISes of $G$. In particular, they are not connected.

Proof. It is enough to prove the statement for vertices adjacent in $T_{G}$. If $x$ belongs to a $k$-DIS $I$ with $y \notin I$, then there should be at least $k$ neighbors of $y$ in $I$ and as $x \in I$, we 
must have $N(x) \cap I=\emptyset$. This implies $|N(y) \backslash N(x)| \geq k$ which contradicts the fact that $x$ and $y$ are almost twins.

If a pair of vertices $x, y \in N(v)$ belong to different components of $T_{G}$ then the $k$-DISes $I$ containing both of $x$ and $y$ are disjoint from $N(x) \cup N(y)$, and $I \backslash\{x, y\}$ should form a $k$-DIS in $G \backslash(N(x) \cup N(y) \cup\{x, y\})$. As $x$ and $y$ are not almost twins, $|N(x) \cup N(y)| \geq \delta+k$ as wlog. $|N(y) \backslash N(x)| \geq k$ and $|N(x)| \geq k$. Thus, the number of $k$-DISes containing both of $x$ and $y$ is at most $m i_{k}(n-\delta-k)$.

On the other hand, if $x$ and $y$ are in the same component $C$ of $T_{G}$, then by Proposition 13 any $k$-DIS $I$ containing both of $x$ and $y$ contains all vertices of $C$, is disjoint from $N(C)$ and $I \backslash C$ is a $k$-DIS in $G \backslash(N(C) \cup C)$ and by the second part of Proposition $13 N(C)$ and $C$ are disjoint. As $|N(C)| \geq \delta$, the number of $k$-DISes containing both of $x$ and $y$ is at most $\operatorname{mi}_{k}(n-\delta-|C|)$.

Writing $s_{1}, s_{2}, \ldots, s_{j}$ for the sizes of the components of $T_{G}$, we obtain

$$
m i_{k}(n) \leq m i_{k}(n-\delta-1)+\frac{\sum_{i=1}^{j}\left(\begin{array}{c}
s_{i} \\
2
\end{array}\right) m i_{k}\left(n-\delta-s_{i}\right)+\left(\left(\begin{array}{c}
\delta \\
2
\end{array}\right)-\sum_{i=1}^{j}\left(\begin{array}{c}
s_{i} \\
2
\end{array}\right)\right) m i_{k}(n-\delta-k)}{\left(\begin{array}{c}
k \\
2
\end{array}\right)}
$$

as every $k$-DIS $I$ with $v \notin I$ was counted at least $\left(\begin{array}{l}k \\ 2\end{array}\right)$ times since $I$ must $k$-dominate $v$.

Let us choose $B=\beta k$ with $\beta=0.8$. This implies $2 \leq B \leq k$ as $k \geq 3$. Suppose that in $T_{G}$ the union of components of size at most $B$ is $s$. Then the number of pairs of vertices within these components is $\sum_{s_{i} \leq B}\left(\begin{array}{c}s_{i} \\ 2\end{array}\right) \leq \frac{s(B-1)}{2}$. Also, the number of pairs within components of size larger than $B$ is $\sum_{s_{i}>B}\left(\begin{array}{c}s_{i} \\ 2\end{array}\right) \leq\left(\begin{array}{c}\delta-s \\ 2\end{array}\right)$. Observe that either $s=\delta$ or $s<\delta-B$.

Observe that $m i_{k}(n-\delta-2) \geq m i_{k}(n-\delta-B) \geq m i_{k}(n-\delta-k)$. Thus majoring all $\left(\begin{array}{l}\delta \\ 2\end{array}\right)$ summands in the following sum we get:

$$
\begin{gathered}
\left.\sum_{i=1}^{j}\left(\begin{array}{c}
s_{i} \\
2
\end{array}\right) m i_{k}\left(n-\delta-s_{i}\right)+\left(\begin{array}{l}
\delta \\
2
\end{array}\right)-\sum_{i=1}^{j}\left(\begin{array}{l}
s_{i} \\
2
\end{array}\right)\right) m i_{k}(n-\delta-k) \leq \\
\leq \sum_{s_{i} \leq B}\left(\begin{array}{c}
s_{i} \\
2
\end{array}\right) m i_{k}(n-\delta-2)+\sum_{s_{i}>B}\left(\begin{array}{c}
s_{i} \\
2
\end{array}\right) m i_{k}(n-\delta-B)+\left(\left(\begin{array}{l}
\delta \\
2
\end{array}\right)-\sum_{i=1}^{j}\left(\begin{array}{c}
s_{i} \\
2
\end{array}\right)\right) m i_{k}(n-\delta-k) \leq \\
\leq \frac{s(B-1)}{2} m i_{k}(n-\delta-2)+\left(\begin{array}{c}
\delta-s \\
2
\end{array}\right) m i_{k}(n-\delta-B)+\left(\left(\begin{array}{l}
\delta \\
2
\end{array}\right)-\frac{s(B-1)}{2}-\left(\begin{array}{c}
\delta-s \\
2
\end{array}\right)\right) m i_{k}(n-\delta-k)
\end{gathered}
$$

As $\left(\begin{array}{l}\delta \\ 2\end{array}\right)=\frac{s(B-1)}{2}+\left[s(\delta-s)+\frac{s(s-B)}{2}\right]+\left(\begin{array}{c}\delta-s \\ 2\end{array}\right)$, this implies that the right hand side of (11) is at most

$$
m i_{k}(n-\delta-1)+\frac{s(B-1)}{2\left(\begin{array}{c}
k \\
2
\end{array}\right)} m i_{k}(n-\delta-2)+\frac{\left(s(\delta-s)+\frac{s(s-B)}{2}\right)}{\left(\begin{array}{c}
k \\
2
\end{array}\right)} m i_{k}(n-\delta-k)+\frac{\left(\begin{array}{c}
\delta-s \\
2
\end{array}\right)}{\left(\begin{array}{c}
k \\
2
\end{array}\right)} m i_{k}(n-\delta-B) .
$$


Recall that we want to prove that $m i_{k}(n) \leq A c^{n}$ for some constant $A$. Using (2), by induction after simplifying it would be enough to show

$$
E:=c^{n}-\left[c^{n-\delta-1}+\frac{s(B-1)}{2\left(\begin{array}{c}
k \\
2
\end{array}\right)} c^{n-\delta-2}+\frac{\left(s(\delta-s)+\frac{s(s-B)}{2}\right)}{\left(\begin{array}{l}
k \\
2
\end{array}\right)} c^{n-\delta-k}+\frac{\left(\begin{array}{c}
\delta-s \\
2
\end{array}\right)}{\left(\begin{array}{c}
k \\
2
\end{array}\right)} c^{n-\delta-B}\right] \geq 0 .
$$

Using that $k \leq \delta$ and simplifying we obtain

$$
\frac{E}{c^{n-\delta-k}} \geq c^{2 k}-\left[c^{k-1}+\frac{s(B-1)}{k(k-1)} c^{k-2}+\frac{s(2 \delta-s-B)}{k(k-1)}+\frac{(\delta-s)(\delta-s-1)}{k(k-1)} c^{k-B}\right] .
$$

We consider two cases, depending on whether $s$ is equal to $\delta$ or not. In the latter case, $s<\delta-B$, as noted already.

Case 2.1: $s=\delta$

In this case, the right hand side of (3) simplifies to

$$
c^{2 k}-c^{k-1}-\frac{\delta(B-1)}{k(k-1)} c^{k-2}-\frac{\delta(\delta-B)}{k(k-1)} .
$$

Since $\delta$ is at most $(1+\varepsilon) k$ and replacing $B$ by $\beta k$, the right hand side of the above inequality is at least

$$
\begin{gathered}
c^{2 k}-c^{k-1}-(1+\varepsilon) \frac{(\beta k-1)}{(k-1)} c^{k-2}-(1+\varepsilon)(1+\varepsilon-\beta)\left(\frac{k}{k-1}\right)=: f_{0}(k, \varepsilon, \beta) \\
\geq c^{2 k}-c^{k}-(1+\varepsilon) \beta c^{k}-(1+\varepsilon)(1+\varepsilon-\beta)\left(1+\frac{1}{1000}\right)=: f_{1}(k, \varepsilon, \beta)
\end{gathered}
$$

for $k>1000$.

Recall that $\varepsilon=0.053$ and $\beta=0.8$. Note that the function $a^{2}-a-(1+\varepsilon) \beta a-(1+$ $\varepsilon)(1+\varepsilon-\beta)\left(1+\frac{1}{1000}\right)$ is increasing in the range $a \geq 1$. At $a=(2+\varepsilon)^{\frac{1}{1+\varepsilon+1 / 1000}}$ the function is positive, thus also for all $k>1000$ at $a=(2+\varepsilon)^{\frac{1}{1+\varepsilon+1 / k}}=c^{k}$ the function is positive. This means $f_{1}(k, \varepsilon, \beta)>0$, which implies $f_{0}(k, \varepsilon, \beta)>0$ for $k>1000$. It is easy to check by a simple computer calculation that $f_{0}(k, \varepsilon, \beta)>0$ for $k \leq 1000$ as well.

Case 2.2: $s<\delta-B$.

Note that $\max _{s<\delta-B}\{s(2 \delta-s-B)\}<(\delta-B) \delta$. Using this, the right hand side of (3) is at least 


$$
\begin{gathered}
c^{2 k}-c^{k-1}-\frac{(\delta-B)(B-1)}{k(k-1)} c^{k-2}-\frac{(\delta-B) \delta}{k(k-1)}-\frac{\delta(\delta-1)}{k(k-1)} c^{k-B} \geq \\
c^{2 k}-c^{k}-(1+\varepsilon-\beta) \frac{(\beta k-1)}{(k-1)} c^{k}-(1+\varepsilon-\beta)(1+\varepsilon) \frac{k}{k-1} \\
-\frac{(1+\varepsilon)(k(1+\varepsilon)-1)}{(k-1)} c^{k-\beta k}:=f_{2}(k, \varepsilon, \beta) \\
\geq c^{2 k}-c^{k}-(1+\varepsilon-\beta) \beta c^{k}-(1+\varepsilon-\beta)(1+\varepsilon+2 / 1000)-(1+\varepsilon)(1+\varepsilon(1+1 / 1000)) c^{(1-\beta) k}:=f_{3}(k, \varepsilon, \beta)
\end{gathered}
$$

for $k>1000$. In the last inequality for bounding the third term we used that $2 / 1000 \geq$ $(1+\varepsilon) /(k-1)$ for $k>1000$ as $\varepsilon=0.053$.

Recall that $\beta=0.8$ and so $1-\beta=\frac{1}{5}$. Observe that the function $a^{10}-a^{5}-(1+\varepsilon-$ $\beta) \beta a^{5}-(1+\varepsilon-\beta)(1+\varepsilon+2 / 1000)-(1+\varepsilon)(1+\varepsilon(1+1 / 1000)) a$ is increasing in $a$ if $a>1$. As for $a=(2+\varepsilon)^{\frac{0.2}{1+\varepsilon+1 / 1000000}}$ the function is positive, also for all $k>1000000$ for the value $a=(2+\varepsilon)^{\frac{0.2}{1+\varepsilon+1 / k}}=c^{k-\beta k}$ the function is positive. This means $f_{3}(k, \varepsilon, \beta)>0$, which implies $f_{2}(k, \varepsilon, \beta)>0$ for $k>1000000$. It is easy to check by a simple computer calculation that $f_{2}(k, \varepsilon, \beta)>0$ for $k \leq 1000000$.

Since $\varepsilon=0.053$ and $c^{k}=(2+\varepsilon)^{\frac{1}{1+\varepsilon+1 / k}} \leq(2+\varepsilon)^{\frac{1}{1+\varepsilon}}$ for any $k \geq 3$, we get $c^{k} \leq 1.98$ for any $k \geq 3$, completing the proof of Theorem 9 .

\section{Acknowledgement}

We are grateful to the MTA Resort Center of Balatonalmádi for their hospitality, where this research was initiated during the Workshop on Graph and Hypergraph Domination in June 2017. We would also like to thank Dániel Soltész for helping us in the optimization process, and for introducing us to Wolframalpha cloud [13].

Research of Gerbner and Patkós was supported by the János Bolyai Research Fellowship of the Hungarian Academy of Sciences.

Research of Gerbner, Keszegh, Methuku and Patkós was supported by the National Research, Development and Innovation Office - NKFIH, grant K 116769.

Research of Patkós and Vizer was supported by the National Research, Development and Innovation Office - NKFIH, grant SNN 116095. 


\section{References}

[1] G. J. Chang and M. J. Jou, Survey on counting maximal independent sets. In: Proceedings of the Second Asian Mathematical Conference, (1995) 265-275.

[2] Z. Füredi, The number of independent sets in connected graphs. J. Graph Theory 11 (1987) 463-470.

[3] J. R. Griggs, C. M. Grinstead, and D. Guichard, The number of maximal independent sets in a connected graph. Discrete Mathematics, 68(2-3) (1988) 211-220.

[4] M. Hujter and Zs. Tuza, The number of maximal independent sets in triangle-free graphs. SIAM J. Discrete Math., 6 (1993) 284-288.

[5] J. W. Moon and L. Moser, On cliques in graphs. Israel J. Math. 3 (1965) 23-28.

[6] O. Morgenstern and J. Von Neumann, Theory of games and economic behavior. Princeton university press, (1945).

[7] Z. L. Nagy, Generalizing Erdős, Moon and Moser's result - The number of $k$-dominating independent sets. Electronic Notes in Discrete Mathematics, proceedings of Eurocomb'17, 61 (2017) 909-915.

[8] Z. L. Nagy, On the Number of $k$-Dominating Independent Sets. Journal of Graph Theory, 84(4) (2017) 566-580.

[9] Problem Booklet of Workshop on Graph and Hypergraph Domination. https://renyi.hu/conferences/graphdom/dominationworkshopbooklet.pdf

[10] B. E. Sagan, A note on independent sets in trees. SIAM Journal on discrete mathematics, 1(1) (1988) 105-108.

[11] H. S. Wilf, The number of maximal independent sets in a tree. SIAM Journal on Algebraic Discrete Methods, 7(1) (1986) 125-130.

[12] A. Włoch, On 2-dominating kernels in graphs. Australas. J. Combin., 53 (2012) 273284.

[13] https://www.wolframcloud.com/

\section{Appendix}

Proposition 14. Suppose $k \geq 3$ is fixed. Then the function

$$
f(\varepsilon)=(2+\varepsilon)^{\frac{1}{1+\varepsilon+1 / k}}
$$

is monotone decreasing in $\varepsilon$ for $\varepsilon \in[0, \infty)$. 
Proof. As $f$ is differentiable, it is enough to prove that the derivative of $f$ is not positive.

$$
f^{\prime}(\varepsilon)=\left(e^{\ln (2+\varepsilon) \frac{1}{1+\varepsilon+\frac{1}{k}}}\right)^{\prime}=(2+\varepsilon)^{\frac{1}{1+\varepsilon+1 / k}}\left(\frac{1}{(2+\varepsilon)\left(1+\varepsilon+\frac{1}{k}\right)}-\frac{\ln (2+\varepsilon)}{\left(1+\varepsilon+\frac{1}{k}\right)^{2}}\right)
$$

So as $(2+\varepsilon)^{\frac{1}{1+\varepsilon+1 / k}} \geq 0$, it is enough to prove that

$$
\frac{1}{(2+\varepsilon)\left(1+\varepsilon+\frac{1}{k}\right)}-\frac{\ln (2+\varepsilon)}{\left(1+\varepsilon+\frac{1}{k}\right)^{2}} \leq 0 .
$$

Simplifying (and using that $1+\varepsilon+\frac{1}{k} \geq 0$ and $2+\varepsilon \geq 0$ ), we get

$$
1+\varepsilon+\frac{1}{k} \leq(2+\varepsilon) \ln (2+\varepsilon)
$$

it is easy to check that for $\varepsilon=0$ the above inequality holds as $k \geq 3$. Now note that the derivative of the right hand side with respect to $\varepsilon$, namely $1+\ln (2+\varepsilon)$, is larger than the derivative of the left hand side, namely 1 . Therefore the above inequality holds for all $\varepsilon \geq 0$, and we are done. 